\title{
TABLE DES MATIERES
}

\author{
ETUDES \\ ENFANTS ET SOCIETTÉS
}

1. SOCIÉTÉ ANTIOUE

Pages

R. ÉTIENNE. - La conscience médicale antique et la vie des enfants ..

Discussion, avec la particlpation de J.P. Bardet, J.-N. BIraben, J. Bourdon,

P. Chaunu, C. Corvisier, R. Dauvergne, $M^{\text {me }}$ Demarolle, J. Dupâquier,

R. Etienne, L. Henry, A. Higounet. .

\section{MOYEN ÂGE}

C. KLAPISCH. - Introduction : Attitudes devant l'enfant $\ldots \quad \ldots \quad \ldots \quad \ldots \quad 63$

A. L'outlllage de l'enfance

F. PIPONNIER. - Les objets de l'enfance $\ldots \begin{array}{lllllllllll} & \ldots & \ldots & \ldots & \ldots & \ldots & \ldots & \ldots & \ldots & & 69\end{array}$

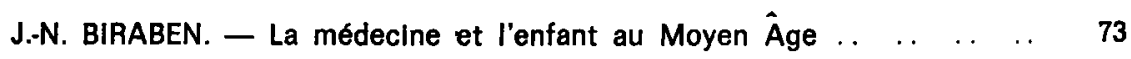

B. La place de l'enfant dans la société

H. ANTONIADIS-BIBICOU. - Quelques notes sur l'enfant de la moyenne

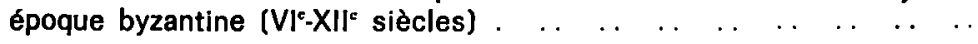

C. PATLAGEAN. - L'enfant et son avenir dans la famille byzantine (IV.

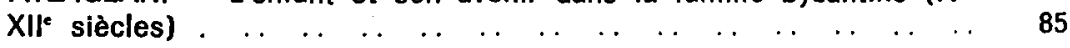

P. RICHÉ. — L'enfant dans le haut Moyen Age $\ldots \begin{array}{llllllllllll} & \ldots & \ldots & \ldots & \ldots & \ldots & \ldots & 95\end{array}$

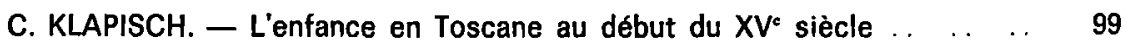

C. Les représentations morales et littéraires

J. BATANY. - Regards sur l'enfance dans la littérature moralisante . . . 123

J. LE GOFF. - Petits enfants dans la littérature des XII-XIIF siècles .. 129

A. TENENTI. - Témoignages toscans sur la mort des enfants autour de $1400 \quad 133$

F. GARNIER. - L'iconographie de l'enfant au Moyen Âge . . . . . . $\quad$. 135

F. BONNEY. - Jean Gerson : un nouveau regard sur l'enfance . . . . . 137

\section{ATTITUDE DE L'ÉGLISE}

J.-L. FLANDRIN. - L'attitude à l'égard du petit enfant et les conduites sexuelles dans la civilisation occidentale : structures anciennes et évolution. 
4. PÉRIODE MODERNE

P. ARIES. - Rapport introductif $\ldots \begin{array}{lllllllllll} & \ldots & \ldots & \ldots & \ldots & \ldots & \ldots & \ldots & \ldots & \ldots & \ldots\end{array}$

J.-N. BIRABEN. - Le médecin et l'enfant au XVIII' siècle (Aperçu sur la

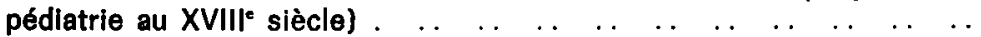

E. HÉLIN. - Une sollicitude ambiguë : l'évacuation des enfants aban-

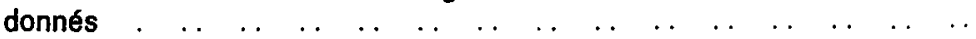

J.-M. GOUESSE. - En Basse-Normandie auX XVII et XVIII siècies : le refus de l'enfant au tribunal de la pénitence $\ldots$.

A. CHAMOUX. - L'enfance abandonnée à Reims à la fin du XVIII siècle.

Dlscussion, avec la participation de A. Armengaud, J.-P. Bardet, J.-N. Biraben, A. Châtelain, P. Chaunu, A. Corvisier, R.P. de Dainville, P. Dayon, J.-L. Flandrin, J.-M. Gouesse, D. Julia, F. Lebrun, J. Lecuir, E. Le Roy Ladurie, R. Mandrou, J.-P. Poussou $\ldots$. .

\section{XIX` SIËCLE}

A. ARMENGAUD. - L'attitude de la société à l'égard de l'enfant au

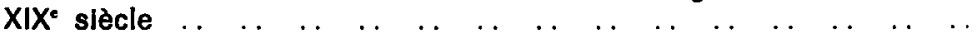

P. LASLETT. - L'attitude à l'égard de l'enfant dans l'Angleterre du $\mathrm{XIX}$ siècle, d'après les sources littéraires, politiques et juridiques ..

C. FOHLEN. - Révolution industrielle et travail des enfants . . . . . . . 319

J.P. KINTZ. - Intervention lors de la réunion du 5 février $1972 \ldots \ldots$. . $\quad \ldots \quad 326$

A. CORVISIER. - La société militaire et l'enfant $\ldots$.

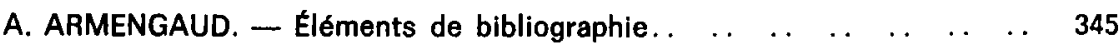

\section{COMPTES RENDUS}

J.P. BARDET. - M. Terrisse, La population de Marseille et de son terroir de 1694 à 1830

A. HIGOUNET-NADAL. - J. Lafon, Régimes matrimoniaux et mutations sociales : Les époux bordelais (1450-1550). Cuadernos de Historia de Espana.

M. LACHIVER. - M. Garden, Lyon et les Lyonnais au XVIII' siècle. M. El Kordi, Bayeux aux XVII' et XVIII' siècles. Contribution à l'histoire urbaine de la France. Commission d'histoire économique et sociale de la Révolution française, Contributions à l'histolre démographique de la Révolution francaise

\section{POSITION DE THESE}

M.T. FONTANILLE. - M.-T. Fontanille, Avortement et contraception dans la médecine gréco-romaino.

\section{DOCUMENTS}

Du soin extrême qu'on doit avoir du baptême des enfants dans le cas d'une fausse couche, ou de la mort d'une femme enceinte (1764), document communiqué par F.-Y. LE MOIGNE ..

Mémoire sur le lait, document communiqué par F.-Y. LE MOIGNE .. . . . . 391 
Remède infaillible contre les vers des enfants, document communiqué par F.-Y. LE MOIGNE

Des hôpitaux d'enfants trouvés, document communiqué par F.Y. LE MOIGNE. 394 Pour que vivent les enfants trouvés, document communiqué par J.P. BARDET. $\quad 395$ Avortement et justice, document communiqué par J.-P. KINTZ .

M. Daquin de Chambéry, Mémoire en réponse aux éclaircissements demandés par la Société royale de médecine de Paris, au sujet des enfants trouvés de Chambery, document communiqué par A. BIDEAU . .

Scène d'accouchement, document communiqué par A. CHAMOUX .. $\quad \ldots \quad \ldots \quad 409$

L'allaltement artificiel, document communiqué par A. CHAMOUX .. $\quad \ldots \quad \ldots \quad \mathbf{4 1 0}$

Mise en nourrice et mortalité des enfants légitimes, document communiqué par A. CHAMOUX ..

Enfants illégitimes et enfants trouvés, document communiqué par $\mathrm{A}$. CHA-

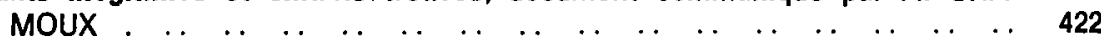

\section{BIBLIOGRAPHIE}

MÉTHODOLOGIE ET GÉNÉRALITÉS

PRÉHISTOIRE - ANTIOUITÉ $\ldots \begin{array}{llllllllllllllll} & \ldots & \ldots & \ldots & \ldots & \ldots & \ldots & \ldots & \ldots & \ldots & \ldots & \ldots & 436\end{array}$

$\begin{array}{lllllllllllllllllll}\text { MOYEN ȦGE } & \ldots & \ldots & \ldots & \ldots & \ldots & \ldots & \ldots & \ldots & \ldots & \ldots & \ldots & \ldots & \ldots & \ldots & \ldots & 437\end{array}$

FRANCE

$\begin{array}{lllllllllllllllll}\text { Généralités } & . & \ldots & \ldots & \ldots & \ldots & \ldots & \ldots & \ldots & \ldots & \ldots & \ldots & \ldots & \ldots & \ldots & \ldots & 439\end{array}$

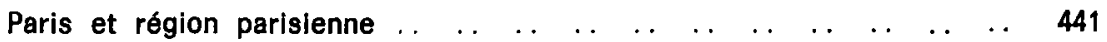

$\begin{array}{lllllllllllllllllll}\text { Nord } & . . & \ldots & \ldots & . & \ldots & \ldots & \ldots & \ldots & \ldots & \ldots & \ldots & \ldots & \ldots & . & . & . & 442\end{array}$

$\begin{array}{lllllllllllllllll}\text { Nord-Est } & \ldots & \ldots & \ldots & \ldots & \ldots & \ldots & \ldots & \ldots & \ldots & \ldots & \ldots & \ldots & \ldots & \ldots & . & 442\end{array}$

$\begin{array}{lllllllllllllllllll}\text { Sud-Est } & \ldots & \ldots & \ldots & \ldots & \ldots & \ldots & \ldots & \ldots & \ldots & \ldots & \ldots & \ldots & \ldots & \ldots & \ldots & 443\end{array}$

$\begin{array}{llllllllllllllllll}\text { Midi } & \ldots & \ldots & \ldots & \ldots & \ldots & \ldots & \ldots & \ldots & \ldots & \ldots & \ldots & \ldots & \ldots & \ldots & \ldots & \ldots & 445\end{array}$

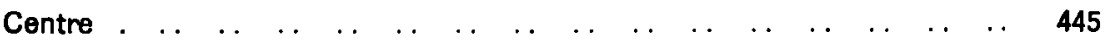

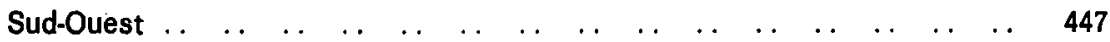

$\begin{array}{lllllllllllllllllll}\text { Ouest } & . & \ldots & \ldots & \ldots & \ldots & \ldots & \ldots & \ldots & \ldots & \ldots & \ldots & \ldots & \ldots & \ldots & \ldots & \ldots & 448\end{array}$

France d'outre-mer .

\section{EUROPE}

$\begin{array}{lllllllllllllllll}\text { Généralités } & . & \ldots & \ldots & \ldots & \ldots & \ldots & \ldots & \ldots & \ldots & \ldots & \ldots & \ldots & \ldots & \ldots & \ldots & 450\end{array}$

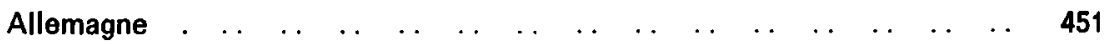

$\begin{array}{llllllllllllllllll}\text { Autriche } & \ldots & \ldots & \ldots & \ldots & \ldots & \ldots & \ldots & \ldots & \ldots & \ldots & \ldots & \ldots & \ldots & \ldots & \ldots & 451\end{array}$

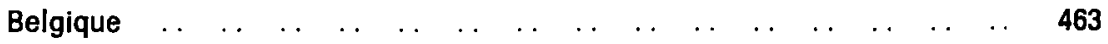

$\begin{array}{lllllllllllllllll}\text { Danemark } & . & \ldots & \ldots & \ldots & \ldots & \ldots & \ldots & \ldots & \ldots & \ldots & \ldots & \ldots & \ldots & \ldots & \ldots & 463\end{array}$

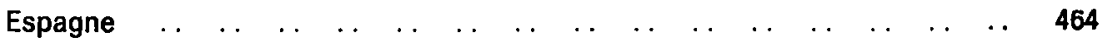

$\begin{array}{lllllllllllllll}\text { Grande-Bretagne } & \ldots & \ldots & \ldots & \ldots & \ldots & \ldots & \ldots & \ldots & \ldots & \ldots & \ldots & \ldots & \ldots & 463\end{array}$

$\begin{array}{lllllllllllllllll}\text { Hongrie } & \ldots & \ldots & \ldots & \ldots & \ldots & \ldots & \ldots & \ldots & \ldots & \ldots & \ldots & \ldots & \ldots & \ldots & . & 466\end{array}$ 


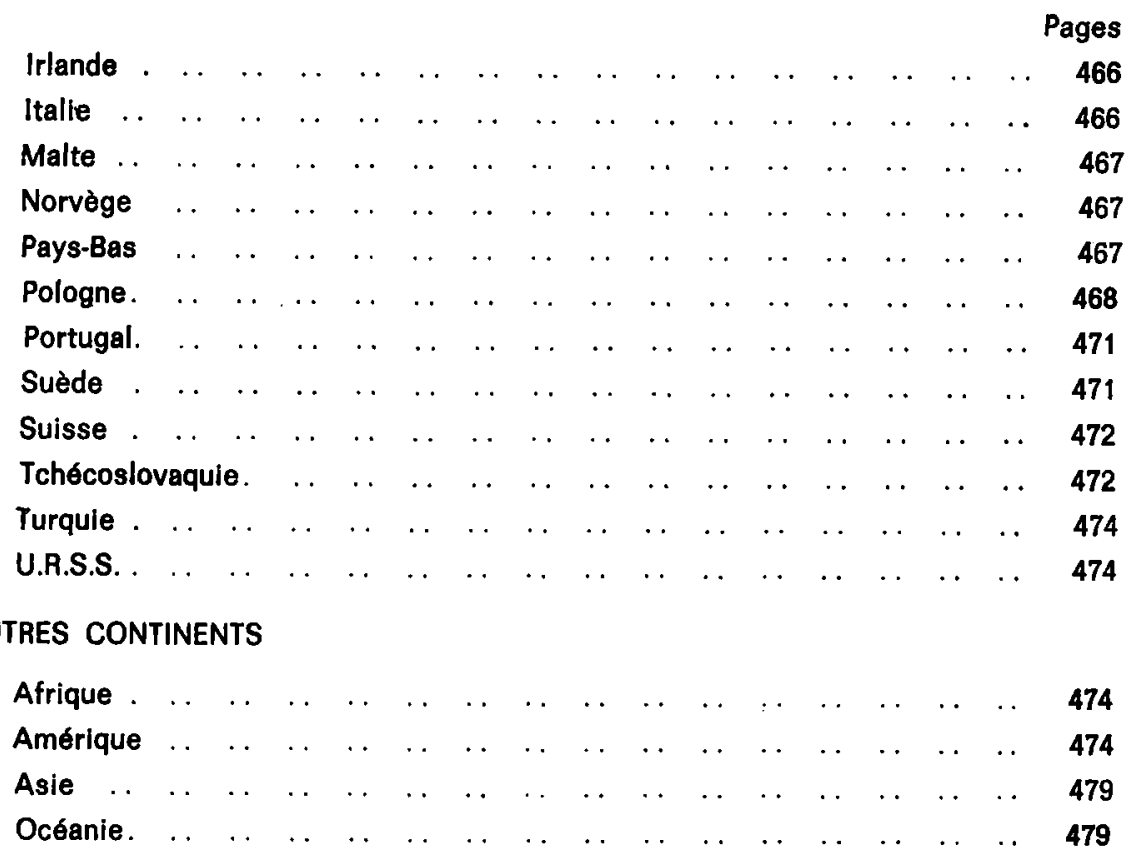

HOMMAGE A MARCEL REINHARD

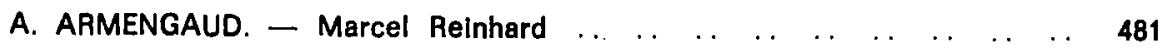

